\title{
EMERGING CONCEPTS IN HIV-ASSOCIATED CRYPTOCOCCAL MENINGITIS
}

Dr David S Lawrence (david.s.lawrence@Ishtm.ac.uk) ${ }^{1,2+}$,

Dr Timothée Boyer-Chammard (timothee.boyer-chammard@pasteur.fr) ${ }^{3,4}$,

Prof Joseph N Jarvis (joseph.jarvis@lshtm.ac.uk)1,2

${ }^{1}$ Botswana-Harvard AIDS Institute Partnership, Private Bag BO 320, Gaborone, Botswana

2 Department of Clinical Research, Faculty of Infectious and Tropical Diseases, London School of Hygiene and Tropical Medicine, Keppel Street, London, WC1E 7HT, UK

${ }^{3}$ Molecular Mycology Unit and National Reference Centre for Invasive Mycoses, Institut Pasteur, Paris, France

${ }^{4}$ Paris Descartes University, Necker Pasteur Centre for Infectious Diseases and Tropical Medicine, Necker Hospital, Paris, France

${ }^{+}$Corresponding author: david.s.lawrence@Ishtm.ac.uk +267 72464834 


\section{ABSTRACT}

Purpose of review: HIV-associated cryptococcal meningitis (CM) remains a significant contributor to AIDS-related mortality despite widened access to antiretroviral therapy. Even in clinical trial settings 10 -week mortality is roughly $40 \%$. A number of important clinical trials have either recently concluded or are actively recruiting.

Recent findings: Global burden of disease etimates suggest CM causes 181,100 deaths annualy. Screening blood for cryptococcal antigen (CrAg) in HIV-infected individuals with CD4 cell counts $<100$ cells $/ \mu \mathrm{L}$ and pre-emptive antifungal treatment for those with detectable $\mathrm{CrAg}$ reduces the incidence of $\mathrm{CM}$ and is likely to reduce mortality. $\mathrm{CM}$ treatment with conventional 14-day courses of amphotericin are associated with high toxicity and mortality and can be reduced to seven days if given alongside flucytosine. Flucytosine is a significantly superior adjunct to amphotericin treatment compared to fluconazole. In settings without amphotericin B dual oral antifungal combinations of flucytosine and fluconazole offer an effective alternative treatment. A single, high-dose of liposomal amphotericin is effective at reducing fungal burden and is being tested in a phase III trial.

Summary: Recently completed and ongoing clinical trials are increasing our understanding of how to optimise induction therapy for CM. Advocacy efforts are needed to broaden access to amphotericin formulations and flucytosine.

Keywords: Cryptococcal meningitis, HIV, AmBisome, Clinical Trial 


\section{INTRODUCTION}

HIV-associated cryptococcal meningitis (CM) remains a significant contributor to AIDSrelated mortality globally, despite roll-out of antiretroviral therapy (ART) (1). Treatment in low and middle income countries (LMICS) is often based on 14-day courses of amphotericin $B$ and fluconazole which is associated with a ten-week mortality of roughly $40 \%(2,3)$. A number of important clinical trials have either recently completed or are currently recruiting participants to help reduce these high mortality rates(2-4). In this paper, we discuss recent trends in the epidemiology of $\mathrm{CM}$, innovation in diagnosis and screening, and discuss the findings of recently completed randomised controlled trials.

\section{EPIDEMIOLOGY}

A recent systematic review examining the global prevalence of cryptococcal antigenaemia ( $\mathrm{CrAg}$ ) in HIV-infected individuals with low CD4 cell counts found a pooled global $\mathrm{CrAg}$ prevalence of $6.5 \%$ in individuals with $C D 4<100$ cells/ $\mu$, and $2 \%$ among those with CD4 counts $101-200$ cells/ $\mu l(5)$. Updated global burden of disease estimates suggest there were approximately 278,000 HIV-infected people positive for $\mathrm{CrAg}$, and an estimated 223,100 incident cases of CM globally in 2014 , with $73 \%$ of these occurring in sub-Saharan Africa. Annual global deaths from CM were estimated at 181,100 (Figure 1)(1). Globally, CM is responsible for roughly $15 \%$ of AIDS-related deaths and is associated with significant longterm disability $(6,7)$.

In Botswana, one of the first countries in sub-Saharan Africa to implement a country-wide ART programme, despite excellent ART coverage there is still a substantial burden of 
advanced HIV, with the 2013-2014 incidence of CM comparable to pre-ART era rates seen in South Africa(8). Data from South Africa also show minimal or no recent decline in the number of CM cases with widespread ART roll out(9). This is likely to result from increasing numbers of patients on long-term ART interrupting, stopping, or failing therapy, offsetting any decline in the numbers of patients presenting for the first time with advanced HIVdisease. The number of patients in South Africa with a CD4 $<100$ cells/uL and the proportion of those who are CrAg positive does not appear to have changed in recent years, however $51 \%$ of patients with a CD4 $<50$ cells/uL are now ART experienced $(9,10)$. Similarly, in Kampala, Uganda, 59\% of patients presenting with CM are now ART experienced (11). These studies highlight the growing complexity of CM patients who often have ART resistance and difficulty with adherence.

\section{DIAGNOSTICS}

Although the gold standard for CM diagnosis remains a positive cryptococcus CSF culture, the current mainstay of cryptococcal diagnosis is the detection of $\mathrm{CrAg}$, a polysaccharide of the cryptococcal capsule which is shed in CSF and blood (Figure 2). CM diagnosis has been revolutionised by the widespread availability of reliable point-of-care Lateral Flow Assays (LFAs) which detect CrAg. The IMMY LFA remains the most sensitive and specific rapid diagnostic test (RDT) available(12). A potential substitute to the IMMY LFA, the StrongStep, was found to have low sensitivity when used on plasma(12). A recently developed RDT, the semi-quantitative Biosynex/BioRad Crypto PS test, incorporates an additional positive line aimed to detect high titers of $\mathrm{CrAg}$ in both blood and CSF. Preliminary results from Cameroon found this test to have comparable performance to the IMMY LFA for antigen screening(13), however further validation in larger studies is needed. 
In areas where CrAg tests are not available the alternative rapid test is India Ink staining of CSF, but sensitivity is around 70\%(14). A study in Uganda has shown that acridine orange, which stains fungal nucleic acids rather than the capsule, was more sensitive than India ink (96\% vs 69\%) with reference to $\mathrm{CrAg}(14)$.

Cryptococcal colony forming units (CFUs) can be counted on serially diluted samples of CSF to enable the quantification of fungal burden, and have been shown to be a strong prognostic indicator(15). However, such quantitative cultures are time consuming and difficult to perform, hence alternatives methods to rapidly assess fungal burden are needed. Trypan blue staining and automated cell counting using the TC20 automated cell counter has been evaluated in Uganda, but found to be poorly predictive of quantitative CFU counts (16). Flow cytometry using a BD LSRFortessa flow cytometer did provide a rapid and accurate measurement of fungal burden in patients with $\mathrm{CM}$ in a small pilot study in South Africa(17), providing proof or principle for the technique, and if combined with a cryptococcal viability stain(18), could potentially be used to assess the response to treatment. Another alternative to quantitative culture is a quantitative polymerase chain reaction (PCR) test for cryptococcus in both blood and CSF being developed by researchers at the Institut Pasteur.

\section{PREVENTION}

CrAg can be detected in the blood many weeks before the onset of CM symptoms(19). WHO guidelines recommended $\mathrm{CrAg}$ screening as the preferred CM prevention strategy in all patients with a CD4 $<100$ cells $/ \mu \mathrm{L}(20)$. Patients who test positive for serum $\mathrm{CrAg}$ should be 
screened for symptoms of $\mathrm{CM}$, and ideally offered a lumbar puncture even in the absence of meningitis symptoms. In cases where patients decline a lumbar puncture, and for all patients without evidence of meningitis, high-dose pre-emptive fluconazole (800$1200 \mathrm{mg} /$ day) is recommended for two weeks followed by standard fluconazole consolidation and maintenance treatment(20). The importance of performing CSF analysis to identify patients in need of more intensive treatment was highlighted in a recent South frican study showing that $34 \%$ of asymptomatic $\mathrm{CrAg}$ positive patients had $\mathrm{CM}(21)$. A systematic review and meta-analysis of CrAg screening studies assessing the prevalence of asymptomatic $\mathrm{CM}$ in $\mathrm{CrAg}$ positive patients, the incidence of $\mathrm{CM}$, and all-cause mortality rates, reported a pooled prevalence of asymptomatic CM in $\mathrm{CrAg}$ positive participants of $33 \%$ (95\% Cl, 21-45\%). The incidence of $\mathrm{CM}$ in $\mathrm{CrAg}$ positive individuals was $21.4 \%(95 \% \mathrm{Cl}$, 11.6-34.4\%) without preemptive fluconazole and $5.7 \%(95 \% \mathrm{Cl}, 3.0-9.7 \%)$ with preemptive fluconazole therapy initiated at $800 \mathrm{mg} / \operatorname{day}(22)$.

National CrAg screening programmes have now been implemented in several African countries, with economic analyses suggesting the intervention should be cost-effective in African settings $(23,24)$. However, early implementation has been challenging, with low rates of uptake when testing is left to the discretion of individual clinicians(25), suggesting automatic, or "reflex" screening of CD4 samples with low CD4 counts is preferable. Even with reflex screening strategies, losses to follow-up or delays in receiving and acting on $\mathrm{CrAg}$ results can adversely impact the effectiveness of the intervention(26). Point of care $\mathrm{CrAg}$ testing may avoid these losses and delays(27), and the IMMY CrAg assay can be performed effectively on finger prick blood samples(28). In settings where CrAg screening is not possible, primary fluconazole prophylaxis remains an option for $\mathrm{CM}$ prevention in 
individuals with advanced HIV-disease(20), with recent data confirming its effectiveness in reducing the incidence of cryptococcal disease(29).

\section{TREATMENT}

The treatment of HIV-associated CM is formed of three phases. An initial two-week induction phase, an eight-week consolidation phase of fluconazole $800 \mathrm{mg} /$ day and maintenance with fluconazole $200 \mathrm{mg} /$ day until the CD4 count reaches $>200$ cells $/ \mu \mathrm{L}$. Until recently the WHO recommended 14-days of daily intravenous amphotericin B given with oral flucytosine or fluconazole for induction therapy in low and middle income countries (LMICs). The recent Advancing Cryptococcal Meningitis Treatment for Africa (ACTA) trial aimed to determine whether amphotericin can be given for seven rather than 14 days; determine the efficacy of flucytosine compared to fluconazole as the oral partner drug to amphotericin B; and test a dual oral antifungal combination(2). A total of 721 patients were randomised to one of five arms: (i) 14-days of amphotericin B with flucytosine; (ii) 14-days of amphotericin B with fluconazole; (iii) Seven-days of amphotericin B with flucytosine, (iv) Seven-days of amphotericin B with fluconazole; (v) 14-days of flucytosine and fluconazole. Pooled analysis of patients receiving amphotericin B demonstrated that seven days was non-inferior to 14 days in terms of all-cause mortality (Figure 3). Among those taking flucytosine as the partner drug, 7 days of amphotericin B had lower ten-week mortality (24\%, $95 \% \mathrm{Cl}: 16-32 \%)$ compared to 14 -days of amphotericin B plus flucytosine( $38 \%, 95 \% \mathrm{Cl}$ : $29-32 \%$, hazard ratio $0.56,95 \% \mathrm{Cl}: 0.35-0.91)$. Notably, the poorest performing arm of the ACTA trial was seven-days amphotericin B plus fluconazole, so a reduction from 14 to sevendays of amphotericin B is only possible in contexts where flucytosine is available. One week of amphotericin B was associated with less drug-induced toxicity, a reduced need for laboratory monitoring, shorter hospital admissions and fewer adverse effects such as 
anaemia requiring blood transfusions. ACTA also confirmed that flucytosine is a significantly superior partner drug for amphotericin B based treatments, leading to a substantial mortality reduction of $38 \%(95 \% \mathrm{Cl}: 16-55 \%, \mathrm{p}=0.002)$ compared to fluconazole. The oral combination arm (fluconazole and flucytosine) was the second best performing arm with a 10-week mortality of $35 \%(95 \% \mathrm{Cl} 29-41 \%)$. In light of these trial data, and a subsequent Cochrane review(30), updated WHO guidelines recommended first-line induction treatment of seven-days of amphotericin B and flucytosine $100 \mathrm{mg} / \mathrm{kg} /$ day, followed by seven-days of fluconazole $1200 \mathrm{mg} /$ day (Table 1)(20).

In high-income settings, an alternative induction regimen is 14-days of liposomal amphotericin and flucytosine(31). Liposomal amphotericin (L-AmB) is associated with fewer drug induced toxicities than the standard deoxycholate formulation, and well suited for use in short-course induction treatment due to the potential for high dosing made possible by the lower rates of toxicity, the long tissue half-life and its effective penetration into brain tissue(32). The recently completed AMBITION phase II trial was performed with the primary objective of determining the rate of clearance of cryptococcus from the cerebrospinal fluid, the Early Fungicidal Activity (EFA), of three alternative schedules of intermittent high dose L$A m B$ in comparison with standard daily L-AmB(4). Eighty participants were recruited at sites in Botswana and Tanzania and randomised to one of four treatment arms: (i) L-AmB 10 $\mathrm{mg} / \mathrm{kg}$ day one (single dose); (ii) L-AmB $10 \mathrm{mg} / \mathrm{kg}$ day one, L-AmB $5 \mathrm{mg} / \mathrm{kg}$ day three (two doses); (iii) L-AmB $10 \mathrm{mg} / \mathrm{kg}$ day one, L-AmB $5 \mathrm{mg} / \mathrm{kg}$ days three and seven (three doses). The control arm (iv) was standard 14-day L-AmB (3mg/kg/day). All were given with high dose fluconazole $(1200 \mathrm{mg} /$ day). The primary analysis showed that the EFA in all three shortcourse high-dose arms was non-inferior to the control arm. There was no evidence for any 
dose response effect with additional L-AmB doses, suggesting maximal fungicidal activity was achieved with a single $10 \mathrm{mg} / \mathrm{kg}$ dose(33). The AMBITION phase III trial is currently comparing a single, high-dose of L-AmB given with 14-days of flucytosine and fluconazole against the current WHO recommended first-line regimen (ISRCTN: 72509687).

\section{Alternative antifungal agents}

The recently completed adjunctive sertraline for the treatment of HIV-associated cryptococcal meningitis (ASTRO) phase III trial explored the addition of sertraline to standard induction with amphotericin and fluconazole, which had shown some promise in a phase II trial(34). Unfortunately, this trial was stopped after recruiting 460 of a planned 550 patients as there was found to be no impact on mortality(35). In an ongoing search for other drugs that could be repurposed, one research team in Vietnam are currently recruiting patients to a phase II trial adding tamoxifen to the standard induction regimen with results expected in 2019. Researchers in the USA have developed a new investigational fungal Cyp51 inhibitor called VT-1129 which has demonstrated potent in vitro activity against cryptococcus(36). Finally, the antihelmith drug flubendazole has shown potential promise(37), along with multiple calcium channel blockers demonstrating action against cryptococcus(38). To date, no clinical trial has been conducted using these agents.

\section{Steroids}

The CRYPTODEX placebo-controlled trial investigated the role of adjunctive dexamethasone during the first six weeks of $\mathrm{CM}$ treatment and was conducted in six counties across Africa and Asia(3). The trial was stopped after the recruitment of 451 patients at the planned midpoint interim analysis which found that steroids did not reduce mortality, significantly 
increased adverse events and disability, and led to slower rates of decline in EFA. Dexamethasone increased the rate of decline in CSF TNF- $\alpha$, which could be an explanation for the slower fungal clearance and poor outcomes(39). Steroids are therefore not a suitable adjunctive treatment during the induction phase, but continue to play a role in the treatment of CM immune reconstitution inflammatory syndrome (IRIS)(20).

\section{Neurapheresis}

A research group in the USA have recently published a proof of concept using an animal model wherein they filtered cryptococcus out of the CSF of rabbits using a process called neurapheresis(40). This approach is in its infancy but warrants further exploration.

\section{Therapeutic Lumbar Punctures}

Lumbar punctures are also essential for the recognition and effective management of raised intracranial pressure (ICP) which is associated with improved outcomes(41). In many highburden settings manometers are not reliably available. As an alternative in such settings, one study in South Africa found the optimal cut-off value for defining high pressure using a standard $22-\mathrm{G}$ spinal needle is $\geq 40 \mathrm{drops} / \mathrm{min}(42)$. In additional to a shortage of equipment, clinicians must often navigate complex cultural barriers to obtaining CSF samples in circumstances whereby patients refuse LP, either for fear of pain - compounded by limited used of local anaesthetic - or concerns that LPs cause death(43).

\section{ANTIRETROVIRAL THERAPY AND IRIS}

IRIS remains a significant complication of $\mathrm{CM}$. Individuals presenting with a first episode of CM manifesting as an unmasking IRIS may suffer worse outcomes than those who have 
been ART exposed (44). A recent Cochrane review has appraised four trials comparing the impact of early ART (within the first four weeks of antifungal initiation) compared with delayed ART (after four weeks) and has concluded that ART should be delayed until at least four weeks due to a potential higher risk of mortality among early initiators(45).

The specific host and pathogen attributes that lead to the development of CM-IRIS are still not fully understood. Development of CM-IRIS has been strongly associated with high central nervous system (CNS) expression of the chemokines MCP-1(CCL2) and MIP-1 $\alpha(C C L 3)$ at initial CM presentation. It is hypothesised that when immune restoration occurs this leads to an influx of inflammatory cells into the CNS, excessive dysregulated local inflammation, and IRIS $(46,47)$. A recent study has shown lower levels of plasma IgM antibodies to some of the cryptococcal polysaccharide antigens and total plasma IgM in cases of CM-IRIS(48), although the role of antibody-mediated protection in CM remains uncertain. This observation may indicate an important role for antibody-mediated protection during $\mathrm{CM}$, and fit with the overarching hypothesis that a poor initial immune response and subsequent failure of effective immune clearance of cryptococcal antigens are key predisposing factors for IRIS (49).

\section{ADVOCACY}

Increasing access to L-AmB in LMICs will help reduce the drug induced toxicities encountered with conventional amphotericin B. Gilead have recently announced an expansion of their preferential pricing programme for visceral leishmaniasis to include $\mathrm{CM}$ which could have a dramatic impact on mortality(50). In addition the FDA have recently included treatments for $\mathrm{CM}$ in their priorty review voucher scheme which could spur further 
drug development in this field(51). Furthermore, despite the strong evidence supporting the use of flucytosine for the treatment of $\mathrm{CM}$, flucytosine remains unregistered and unavailable in African and Asian LMICs with very few companies manufacturing the drug. It is hoped that with an increasing acknowledgement of the role of flucytosine its production, registration and distribution will broaden(52).

\section{CONCLUSION}

Cryptococcal meningitis continues to pose a major clinical challenge in resource limited settings. Screening and diagnosis have been revolutionised by the highly sensitive lateral flow assay, and seminal clinical trials have improved our understanding of how to optimise induction therapy, with shorter-courses of amphotericin given with flucytosine to reduce mortality rates, and the avoidance of harmful adjunctive steroids. However, advocacy efforts are urgently needed to ensure that patients with $\mathrm{CM}$ have access to these novel diagnostics and treatments so that recent advances in evidence can be translated into clinical practice.

\section{KEY POINTS}

- Cryptococcal meningitis remains a significant cause of AIDS-associated mortality

- Treatment with conventional 14-day courses of amphotericin are associated with high toxicity and mortality and can be reduced to seven days if given alongside flucytosine.

- Flucytosine is a significantly superior adjunct to amphotericin treatment compared to fluconazole. 
- A single, high-dose of liposomal amphotericin is effective at reducing fungal burden and is being tested in a phase III trial.

ACKNOWLEDGEMENTS: None

FINANCIAL SUPPORT AND SPONSORSHIP: This work was supported by the Penn Center for AIDS Research, a National Institutes of Health $(\mathrm{NIH})$-funded program (grant number P30 Al 045008) to JNJ. DSL, TBC and JNJ are all investigators on the AMBITION trial which is jointly funded through the European Developing Countries Clinical Trials Partnership (EDCTP), the Swedish International Development Cooperation Agency (SIDA), and the Wellcome Trust / Medical Research Council (UK) / UKAID Joint Global Health Trials.

CONFLICTS OF INTEREST: JNJ was the recipient of a Gilead Investigator Initiated Award (completed). DSL, TBC and JNJ are all investigators on the AMBITION trial. 


\section{REFERENCES}

1. Rajasingham R, Smith RM, Park BJ, Jarvis JN, Govender NP, Chiller TM, et al. Global burden of disease of HIV-associated cryptococcal meningitis: an updated analysis. Lancet Infect Dis. 2017;17(8):873-81.

* An updated global burden of disease study which demonstrates the ongoing high rates of cryptococcal antigenaemia in patients with low CD4 counts and the number of cases of cryptococcal meningitis and associated mortality.

2. Molloy SF, Kanyama C, Heyderman RS, Loyse A, Kouanfack C, Chanda D, et al. Antifungal Combinations for Treatment of Cryptococcal Meningitis in Africa. N Engl J Med. 2018;378(11):1004-17.

** A large phase III randomised controlled trial which found one week of amphotericin B given with flucytosine to be non-inferior to two, flucytosine to be superior to fluconazole and for dual oral antifungal treatment with flucytosine and fluconazole to be a suitable alternative to intravenous treatment.

3. Beardsley J, Wolbers M, Kibengo FM, Ggayi AB, Kamali A, Cuc NT, et al. Adjunctive Dexamethasone in HIV-Associated Cryptococcal Meningitis. N Engl J Med. 2016;374(6):54254.

**This phase III randomised controlled trial found that despite adjunctive dexamethose during the first six-week of treatment having an impact on cerebrospinal fluid opening pressure it had no impact on mortality, slowed the rate of fungal clearance from the brain and was associated with more adverse events and higher rates of diasbility.

4. Jarvis J, Leeme T, Molefi M, Chofle AA, Bidwell G, Tsholo K, et al. Short Course Highdose Liposomal Amphotericin B for HIV-associated Cryptococcal Meningitis: A phase-II Randomized Controlled Trial. CID. 2018; published online June 26, 2018.

**This phase II randomised controlled trial has demonstrated that a single, high-dose of liposomal amphotericin is non-inferior to 14-days of daily dosed treatment at clearing cryptococcus from the brain. This group have now commenced recruitment within a large, phase III trial with all-cause mortality as the primary outcome.

5. Ford N, Shubber Z, Jarvis JN, Chiller T, Greene G, Migone C, et al. CD4 Cell Count Threshold for Cryptococcal Antigen Screening of HIV-Infected Individuals: A Systematic Review and Meta-analysis. Clin Infect Dis. 2018;66(suppl_2):S152-s9.

6. Pasquier E, Kunda J, De Beaudrap P, Loyse A, Temfack E, Molloy SF, et al. Long-term Mortality and Disability in Cryptococcal Meningitis: A Systematic Literature Review. Clin Infect Dis. 2018;66(7):1122-32. 
7. Goldberg DW, Tenforde MW, Mitchell HK, Jarvis JN. Neurological Sequelae of Adult Meningitis in Africa: A Systematic Literature Review. Open Forum Infect Dis. 2018;5(1):ofx246.

8. Tenforde MW, Mokomane M, Leeme T, Patel RKK, Lekwape N, Ramodimoosi C, et al. Advanced Human Immunodeficiency Virus Disease in Botswana Following Successful Antiretroviral Therapy Rollout: Incidence of and Temporal Trends in Cryptococcal Meningitis. Clin Infect Dis. 2017;65(5):779-86.

* A paper demonstrating that despite widened access to antiretrovial therapy there are similar numbers of cases of cryptococcal meningitis seen now than in the pre-treatment era. 9. Osler M, Hilderbrand K, Goemaere E, Ford N, Smith M, Meintjes G, et al. The Continuing Burden of Advanced HIV Disease Over 10 Years of Increasing Antiretroviral Therapy Coverage in South Africa. Clin Infect Dis. 2018;66(suppl_2):S118-s25.

10. Coetzee LM, Cassim N, Sriruttan C, Mhlanga M, Govender NP, Glencross DK. Cryptococcal antigen positivity combined with the percentage of HIV-seropositive samples with CD4 counts $<100$ cells/mul identifies districts in South Africa with advanced burden of disease. PLoS One. 2018;13(6):e0198993.

11. Flynn AG, Meya DB, Hullsiek KH, Rhein J, Williams DA, Musubire A, et al. Evolving Failures in the Delivery of Human Immunodeficiency Virus Care: Lessons From a Ugandan Meningitis Cohort 2006-2016. Open Forum Infect Dis. 2017;4(2):ofx077.

12. Mpoza E, Mukaremera L, Kundura DA, Akampurira A, Luggya T, Tadeo KK, et al. Evaluation of a point-of-care immunoassay test kit 'StrongStep' for cryptococcal antigen detection. PLoS One. 2018;13(1):e0190652.

13. Temfack E, Kouanfack C, Mossiang L, Loyse A, Fonkoua MC, Molloy SF, et al. Cryptococcal Antigen Screening in Asymptomatic HIV-Infected Antiretroviral Naive Patients in Cameroon and Evaluation of the New Semi-Quantitative Biosynex CryptoPS Test. Front Microbiol. 2018;9:409.

14. Kwizera R, Akampurira A, Williams D, Boulware DR, Meya DB. Acridine orange fluorescent microscopy is more sensitive than India ink light microscopy in the rapid detection of cryptococcosis among CrAg positive HIV patients. PLoS One.

2017;12(7):e0182108.

15. Jarvis JN, Bicanic T, Loyse A, Namarika D, Jackson A, Nussbaum JC, et al.

Determinants of mortality in a combined cohort of 501 patients with HIV-associated Cryptococcal meningitis: implications for improving outcomes. Clin Infect Dis. 2014;58(5):736-45.

16. Kwizera R, Akampurira A, Kandole TK, Nabaggala MS, Williams DA, Kambugu A, et al. Evaluation of trypan blue stain in the TC20 automated cell counter as a point-of-care for the enumeration of viable cryptococcal cells in cerebrospinal fluid. Med Mycol. 2018;56(5):55964.

17. Scriven JE, Graham LM, Schutz C, Scriba TJ, Wilkinson RJ, Boulware DR, et al. Flow Cytometry To Assess Cerebrospinal Fluid Fungal Burden in Cryptococcal Meningitis. J Clin Microbiol. 2016;54(3):802-4.

18. McMullan BJ, Desmarini D, Djordjevic JT, Chen SC, Roper M, Sorrell TC. Rapid microscopy and use of vital dyes: potential to determine viability of Cryptococcus neoformans in the clinical laboratory. PLoS One. 2015;10(1):e0117186.

19. Jarvis JN, Lawn SD, Vogt M, Bangani N, Wood R, Harrison TS. Screening for cryptococcal antigenemia in patients accessing an antiretroviral treatment program in South Africa. Clin Infect Dis. 2009;48(7):856-62. 
20. WHO. Guidelines for the diagnosis, prevention, and management of cryptococcal disease in HIV-infected adults, adolescents and children. Geneva: World Health Organisation; 2018 March 2018.

** Updated WHO guidelines with significant changes to treatment recommendations in low and middle income countries reflecting the findings of the ACTA trial.

21. Wake RM, Britz E, Sriruttan C, Rukasha I, Omar T, Spencer DC, et al. High Cryptococcal Antigen Titers in Blood are Predictive of Subclinical Cryptococcal Meningitis Among HIV-Infected Patients. Clin Infect Dis. 2017.

*This study highlights the need for cryptococcal antigen screening and the importance of identifying, investigating and treating those with a positive test to avoid development of cryptococcal meningitis.

22. Temfack E, Bigna JJ, Luma HN, Spijker R, Meintjes G, Jarvis JN, et al. Impact of routine cryptococcal antigen screening and targeted pre-emptive fluconazole therapy in antiretroviral naive HIV-infected adults with less than 100 CD4 cells/muL: a systematic review and meta-analysis. Clin Infect Dis. 2018.

23. Ramachandran A, Manabe $Y$, Rajasingham R, Shah M. Cost-effectiveness of CRAGLFA screening for cryptococcal meningitis among people living with HIV in Uganda. BMC Infect Dis. 2017;17(1):225.

24. Larson BA, Rockers PC, Bonawitz R, Sriruttan C, Glencross DK, Cassim N, et al. Screening HIV-Infected Patients with Low CD4 Counts for Cryptococcal Antigenemia prior to Initiation of Antiretroviral Therapy: Cost Effectiveness of Alternative Screening Strategies in South Africa. PLoS One. 2016;11(7):e0158986.

25. Vallabhaneni S, Longley N, Smith M, Smith R, Osler M, Kelly N, et al. Implementation and Operational Research: Evaluation of a Public-Sector, Provider-Initiated Cryptococcal Antigen Screening and Treatment Program, Western Cape, South Africa. J Acquir Immune Defic Syndr. 2016;72(2):e37-e42.

26. Govender NP, Roy M, Mendes JF, Zulu TG, Chiller TM, Karstaedt AS. Evaluation of screening and treatment of cryptococcal antigenaemia among HIV-infected persons in Soweto, South Africa. HIV Med. 2015;16(8):468-76.

27. Wake RM, Glencross DK, Sriruttan C, Harrison TS, Govender NP. Cryptococcal antigen screening in HIV-infected adults: let's get straight to the point. Aids. 2016;30(3):339-42.

28. Wake RM, Jarvis JN, Harrison TS, Govender NP. Brief Report: Point of Care Cryptococcal Antigen Screening: Pipetting Finger-Prick Blood Improves Performance of Immunomycologics Lateral Flow Assay. J Acquir Immune Defic Syndr. 2018;78(5):574-8.

29. Hakim J, Musiime V, Szubert AJ, Mallewa J, Siika A, Agutu C, et al. Enhanced Prophylaxis plus Antiretroviral Therapy for Advanced HIV Infection in Africa. N Engl J Med. 2017;377(3):233-45.

30. Tenforde MW, Shapiro AE, Rouse B, Jarvis JN, Li T, Eshun-Wilson I, et al. Treatment for HIV-associated cryptococcal meningitis. Cochrane Database Syst Rev. 2018;7:Cd005647.

*This Cochrane review has appraised all completed clinical trials providing different antifungal treatment regimens to reflect recently acquired evidence and support the updating of WHO guidelines.

31. Nelson M, Dockrell D, Edwards S, Angus B, Barton S, Beeching N, et al. British HIV Association and British Infection Association guidelines for the treatment of opportunistic infection in HIV-seropositive individuals 2011. HIV Med. 2011;12 Suppl 2:1-140. 
32. Lestner J, McEntee L, Johnson A, Livermore J, Whalley S, Schwartz J, et al.

Experimental Models of Short Courses of Liposomal Amphotericin B for Induction Therapy for Cryptococcal Meningitis. Antimicrob Agents Chemother. 2017;61(6).

33. Bicanic T, Bottomley C, Loyse A, Brouwer AE, Muzoora C, Taseera K, et al. Toxicity of Amphotericin B Deoxycholate-Based Induction Therapy in Patients with HIV-Associated Cryptococcal Meningitis. Antimicrob Agents Chemother. 2015;59(12):7224-31.

34. Rhein J, Morawski BM, Hullsiek KH, Nabeta HW, Kiggundu R, Tugume L, et al. Efficacy of adjunctive sertraline for the treatment of HIV-associated cryptococcal meningitis: an open-label dose-ranging study. Lancet Infect Dis. 2016;16(7):809-18.

35. Rhein J, Hullsiek KH, Tugume L, Nuwagira E, Mpoza E, Kiggundu R, et al. Adjunctive sertaline in HIV-associated cryptococcal meningitis. CROI; March 4-7 2018; Boston, Massachusetts2018.

**This oral presentation outlined the findings of the ASTRO study which found that adjunctive sertraline during induction treatment for cryptococcal meningitis had no impact on mortality.

36. Lockhart SR, Fothergill AW, Iqbal N, Bolden CB, Grossman NT, Garvey EP, et al. The Investigational Fungal Cyp51 Inhibitor VT-1129 Demonstrates Potent In Vitro Activity against Cryptococcus neoformans and Cryptococcus gattii. Antimicrob Agents Chemother. 2016;60(4):2528-31.

37. Nixon GL, McEntee L, Johnson A, Farrington N, Whalley S, Livermore J, et al. Repurposing and Reformulation of the Antiparasitic Agent Flubendazole for Treatment of Cryptococcal Meningoencephalitis, a Neglected Fungal Disease. Antimicrob Agents Chemother. 2018;62(4).

38. Truong M, Monahan LG, Carter DA, Charles IG. Repurposing drugs to fast-track therapeutic agents for the treatment of cryptococcosis. PeerJ. 2018;6:e4761.

39. Beardsley J, Hoang NLT, Kibengo FM, Tung NLN, Binh TQ, Hung LQ, et al. Do intracerebral cytokine responses explain the harmful effects of dexamethasone in HIV-associated cryptococcal meningitis? Clin Infect Dis. 2018.

40. Smilnak GJ, Charalambous LT, Cutshaw D, Premji AM, Giamberardino CD, Ballard CG, et al. Novel Treatment of Cryptococcal Meningitis via Neurapheresis Therapy. J Infect Dis. 2018;218(7):1147-54.

*This interesting proof of concept study used a process called neurapheresis to filter the circulating cerebrospinal fluid of rabbits infected with cryptococcus.

41. Rolfes MA, Hullsiek KH, Rhein J, Nabeta HW, Taseera K, Schutz C, et al. The effect of therapeutic lumbar punctures on acute mortality from cryptococcal meningitis. Clin Infect Dis. 2014;59(11):1607-14.

42. Boyles TH, Gatley E, Wasserman S, Meintjes G. Brief Report: Flow Rate of Cerebrospinal Fluid Through a Spinal Needle Can Accurately Predict Intracranial Pressure in Cryptococcal Meningitis. J Acquir Immune Defic Syndr. 2017;74(3):e64-e6.

43. Thakur KT, Mateyo K, Hachaambwa L, Kayamba V, Mallewa M, Mallewa J, et al. Lumbar puncture refusal in sub-Saharan Africa: A call for further understanding and intervention. Neurology. 2015;84(19):1988-90.

44. Rhein J, Hullsiek KH, Evans EE, Tugume L, Nuwagira E, Ssebambulidde K, et al. Detrimental Outcomes of Unmasking Cryptococcal Meningitis With Recent ART Initiation Open Forum Infectious Diseases. 2018;5(8). 
45. Eshun-Wilson I, Okwen MP, Richardson M, Bicanic T. Early versus delayed antiretroviral treatment in HIV-positive people with cryptococcal meningitis. Cochrane Database Syst Rev. 2018;7:Cd009012.

**Updated Cochrane review reaffirming the need to delay antiretroviral therapy initiation for at least four weeks from initiation of antifungal treatment for cryptococcal meningitis. 46. Chang CC, Omarjee S, Lim A, Spelman T, Gosnell BI, Carr WH, et al. Chemokine levels and chemokine receptor expression in the blood and the cerebrospinal fluid of HIV-infected patients with cryptococcal meningitis and cryptococcosis-associated immune reconstitution inflammatory syndrome. J Infect Dis. 2013;208(10):1604-12.

47. Jarvis JN, Meintjes G, Bicanic T, Buffa V, Hogan L, Mo S, et al. Cerebrospinal fluid cytokine profiles predict risk of early mortality and immune reconstitution inflammatory syndrome in HIV-associated cryptococcal meningitis. PLoS Pathog. 2015;11(4):e1004754. 48. Yoon HA, Nakouzi A, Chang CC, Kuniholm MH, Carreno LJ, Wang T, et al. Association between plasma antibody responses and risk for Cryptococcus-associated immune reconstitution inflammatory syndrome. J Infect Dis. 2018.

49. Jarvis JN, Harrison TS. Understanding Causal Pathways in Cryptococcal Meningitis Immune Reconstitution Inflammatory Syndrome. J Infect Dis. 2018.

50. Gilead Sciences Announces Steep Discounts for Ambisome to Treat Cryptococcal Meningitis in Low - and Middle-Income Countries [press release]. Internet, September 7th 20182018.

51. FDA adds four tropical diseases to priority review voucher program to encourage drug development in areas of unmet need [press release]. Internet, August 23rd 20182018. 52. Molloy SF, Chiller T, Greene GS, Burry J, Govender NP, Kanyama C, et al. Cryptococcal meningitis: A neglected NTD? PLoS Negl Trop Dis. 2017;11(6):e0005575. 
Figure 1 (previously published): Estimated annual incidence of cryptococcal infection by country in $2014(1)$

Figure 2 (original): Negative and positive cryptococcal antigen lateral flow assay (left), cryptococcal culture on Sabouraud agar medium (top right) and India ink staining showing cryptococcus in CSF (lower right).

Figure 3 (previously published): Cumulative all cause mortality from the ACTA trial(2).

A: Cumulative all-cause mortality by week 10 according to treatment arm.

B: Comparison of cumulative all-cause mortality by week 10 between flucytosine and fluconazole as amphotericin B partner treatment

C: Cumulative all-cause mortality by week 10 according to the treatment strategy

Table 1 (original): WHO 2018 guidelines for management of cryptococcal disease in HIVinfected adults (20). 